\title{
Improving Quality of Services in Maternity Care Triage System
}

\author{
António Abelha ${ }^{a}$, Eliana Pereira ${ }^{a}$, Andreia Brandão ${ }^{a}$, Filipe Portela ${ }^{b}$, Manuel Filipe \\ Santos $^{b}$, José Machado ${ }^{a}$, Jorge Braga ${ }^{c}$ \\ ${ }^{a}$ Computer Science and Technology Center (CCTC). University of Minho. Braga. \\ Portugal; \\ ${ }^{b}$ Algoritmi Research Center. University of Minho. Guimarães. Portugal; \\ ${ }^{c}$ Centro Materno Infantil do Norte, Centro Hospitalar do Porto, Porto, Portugal \\ abelha@di.uminho.pt; a60196@alunos.uminho.pt; a58549@alunos.uminho.pt; \\ cfp@dsi.uminho.pt; mfs@dsi.uminho.pt; jmac@di.uminho.pt; \\ jorgesousabraga@gmail.com
}

\begin{abstract}
The main objectives in hospital triages are to improve the quality of care and reduce the risks associated to the waiting time in emergency care. Thus, an efficient triage is a good way to avoid some future problems and how much quicker it is, more the patient can benefit. The most common triage system is the Manchester Triage System (MTS). MTS is a reliable system focused in the hospital emergency department. However, its use is more suitable for more widespread medical emergencies and not for specialized cases as is Maternity Care and Gynecological and Obstetrics emergencies. To overcome these limitations an alternative pre-triage system, integrated into an intelligent decision support system, was developed in order to better characterize the patient and correctly defined the patient as urgent or not. This system allows increasing patient's safety, especially women who need immediate care. This paper presents the main results and the workflow describing the decision process in real time in the emergency department, when the women are submitted to the triage process and they are identified possible evolution points of this system.
\end{abstract}




\section{Keywords}

Triage; Emergency Department; Intelligent Decision Support System; Manchester Triage System; Triage System Gynecology Obstetrics; Real Time Decision Support

\section{Introduction}

When a patient is admitted at a medical emergency department, he is submitted to a triage process in order to determine the priority of treatment based on their clinical status. The priority of attendance is determined taking into account the patient clinical condition. To this end, in the hospital might be used several types of triage systems. The most commonly used are those classification systems with five levels of severity, the Emergency Severity Index (ESI) (Murray et al., 2004) and the Canadian Triage and Acuity Scale (CTAS) (Beveridge R., 1998) (Cabral et al., 2011). These systems are efficient when it comes to emergency situations in general, however it reveals little flexibility to specific cases as is Maternity Care. In fact and as quoted by the Portuguese Health General Directorate (Direcção Geral de Saúde (DGS), there is a set of specificities that should have be taken into account in maternity care such as: There are pregnant cared in childbirth or labor physiology which should have different emergency levels. And the fact that there are pregnant cared in diverse situations: pathological, obstetric or medical, that can conditioning the pregnancy with different levels of emergency and care. Faced with a pregnant it is necessary to define and classify the clinical emergency because of having two patients with different pathologies. The Maternity Care is a DGS concern when it refers that any system of triage for adults should be prepared to attend emergency cases of gynecology and obstetrics (GO), following the triage model for Obstetrics published in the DGS site in 2013.

Thus there is a need to develop a priority triage system able to distinguish urgent cases as for example Manchester Triage System (MTS) does. However this new system should be specific to obstetrics and gynecologists cases. This system will be extremely useful because it will triage patients with a higher degree of accuracy. This article presents the first stage of implementing a priority triage system for 
gynecology and obstetrics with five levels of priorities as it happen for example with MTS system. In this stage, this system just are ready to drive the pregnant to emergency (URG) or consult (ARGO), according to their clinical situation. As referred in a previous work (Cabral et al., 2011), today women seeking care for genecology and obstetrics (pregnant, postpartum, non-postpartum, maybe pregnant, for Voluntary Interruption of Pregnancy (VIP) or for Cardiotocography (CTG)) pass through the implemented triage system. This system was developed and implemented in Maternidade Júlio Dinis (MJD) since 2010, then it was extended to Centro Materno Infantil do Norte (CMIN) - new maternity care service, replacing MJD. Although this system was designed and tested in a specific Maternity, it can be deployed in any unit of obstetrics and gynecology. Thus the focus of this article includes a description of the decision support system that sustains the triage service currently implemented in CMIN and presents the possible improvements.

Beyond the introduction, this paper includes six sections. The second one is related to the background knowledge and introduces the CMIN, AIDA, some triage system. The third section presents the Triage System for Gynecology and Obstetrics emergency department. The forth section makes an analysis of the pre-triage system and an exploration of possible developments. Finally, the fifth part of this paper presents the discussion and the conclusion, while the last section presents some future work that will be implemented in this area.

\section{Background and Related Work}

\section{Context}

Maternity care refers to a specific health care treatment given to the pregnancy and delivery of a newborn child. Maternity care is provided on the basis of the physical and psychosocial needs of the patient, the patient's entire family, and the newly born offspring. Often these units also meet health patients in emergency conditions. As is normally observed in the emergency room of general hospitals, the maternity also requires a specific triage system meeting the specific priorities of patients who are 
served by this type of health care institution. In this context, a specific emergency triage systems to maternity care had been developed but they are far away to be a proper system. An ideal emergency triage system should quickly distinguish patients in emergency life-threatening conditions, patients with serious diseases but not in lifethreatening and patients without serious situations. In case of Obstetrics care, should be considered the life of the mother and the fetus. This is the main reason to having difficulties in finding an emergency triage system adequate to Obstetrics. Sometimes light symptoms in women may result in a life threatening to the fetus. In other cases minimal medical conditions are enough but usually are exacerbated by the pregnant woman anxiety. Therefore it is required a triage system capable of detecting these situations, since the Manchester Triage system is not able to make this type of recognition in the Maternity care. This is a common situation in the most general hospitals, as it was proved in Centro Hospitalar do Porto (CHP) - Hospital Santo António (HSA) (hospital used to test the new methodology / approach to triage maternity care patient).

In 2012, they were performed some analysis on the performance of the pre-triage system, evaluating the year 2010 and having in attention the result being URG or ARGO (Cabral \& Abelha, 2012). The results of this analysis are presented in Table 1. For example, in the URG level the waiting time average is around 25 minutes and has a standard deviation of 21 minutes. The waiting time is framed in a temporal limit between less 1 minute and more than 5 hours and 30 minutes.

Table 1 - Statistics about pre-triage system waiting time relative to 2010 (adapted from (Cabral et al. 2011))

\begin{tabular}{|r|r|r|r|r|r|r|r|}
\hline Level & $\begin{array}{c}\text { Number of } \\
\text { patients }\end{array}$ & Average & $\begin{array}{r}\text { Standard } \\
\text { Deviation }\end{array}$ & Mode & Median & Minimum & Maximum \\
\hline URG & 10146 & $0: 24: 40$ & $0: 21: 06$ & $00: 08: 20$ & $00: 17: 07$ & $0: 00: 15$ & $5: 39: 45$ \\
\hline ARGO & 8265 & $0: 24: 23$ & $0: 21: 20$ & $00: 03: 43$ & $00: 16: 01$ & $0: 00: 24$ & $5: 17: 38$ \\
\hline
\end{tabular}

In 2014 it was founded the Centro Materno Infantil do Norte (CMIN) and all services offered in MJD were transferred to CMIN, including the pre-triage system. 


\section{Agency for Integration, Archive and Diffusion of Medical Information}

AIDA (Agency for Integration, Archive and Diffusion of Medical Information) is a platform based on multi-agent system. The main goal is overcomes the difficulties

presented by the uniformity of clinical systems and by the complexity of medical and administrative data provided by different sources of hospital information. This platform presents a proactive behavior and combines a set of functions and features like communication between heterogeneous systems, storage management of hospital information; sending and receiving information from hospital sources, such as laboratories (laboratories) (medical reports, images, recipes, etc.). Due to AIDA features, it allows the interoperability between subsystems of the hospital, taking the lead and having an important role in the hospitals by providing an easy access to information, thereby allowing a clinical information management anywhere in the hospital. In the CMIN all the information are connected through AIDA. AIDA allows to access for example to the clinical data provided by SAPE (Nursing Support System) and SONHO (Clinical Information System) (Abelha et al. 20103; Machado et al. 2007; Cardoso et al. 2014).

\section{Manchester Triage System}

The main method used in the triage process by the professional community of the HSA is the Manchester Triage System (MTS). The MTS is a scale used in the triage process of patients when they are admitted to the Emergency Department (ED). This method was introduced in the UK in 1996, now is already widespread in Europe and began to be implemented in the United States in 2000 (Mackway-Jones K., 1996). This system includes a set of 52 flowcharts, and in each one of them is represented an abuse of a patient. Each flowchart contains discriminators that allow triage nurses direct the patient to a particular category of emergency, which is associated with a maximum wait time until the patient is attended by the doctor. MTS is a very simple system that allows quick access to the clinical status of patients and can be applied to a target population with a broad range of symptoms. However, their use is more suitable for more widespread medical emergencies and not for specialized medical emergencies for women in need of medical assistance in GO (Moll, 2009). 


\section{Obstetric Triage Acuity Scale (OTAS)}

The Obstetric Triage Acuity Scale (OTAS) was molded based on the Canadian Triage and Acuity Scale (CTAS) (Murray et al., 2004). The CTAS has a high degree of reliability and validity. However it includes only a limited number of obstetric determinants that do not reflect the diversity of patients who frequent triage units obstetrics. Thus, in order to enable the creation of a tool that would encompass the wide variety of patients that come in obstetric triage units it was developed the OTAS (Smithson et al., 2013). In general it can be identified the following advantages for the OTAS system: Considers separately the maternal and fetal assessments; Separates labor pathology of pregnancy; Acceptable structured to obstetrics definition signals, on symptoms, timing of care in timing of reassessment. On the other hand the in Portugal, OTAS is not implemented in national health system, so that it does not permit a local review.

\section{Pre-Triage System for Gynecology and Obstetrics}

The health professionals of CHP concluded that MTS was not the most appropriate system for the GO service, due to the degree of generalization of the guidelines. Thus, it was developed a pre-triage system specific for GO based on MTS system. To support a pre-triage system is essential having a platform as is AIDA which allows having access to multiple patient data provided from different data-sources in real-time.

\section{Real-Time Decision Support}

The decision support system is based on a set of predefined questions in the form of rules, representing a decision tree. When a patient is admitted to ED of gynecology and obstetrics, the type of transport used is very important. If she was transported by ambulance, she will be assisted immediately. In case of a woman being transported by another means, the state of pregnancy is evaluated and it is determined whether it needs immediate assistance or be referred to the pre-triage system implemented (GO Triage). In this case, it must wait a few seconds/minutes, depending on the number of patient in urgent care to be called to the triage room. In the triage room, she will answer a specific 
questionnaire for GO normally performed by the nurses. This process is fully electronic and is supported by AIDA platform. Most of the question are answered as yes (1) or no (0). In the figure 1 is described the flowchart that attempts to map the triage questionnaire which supports the process of decision-making system.

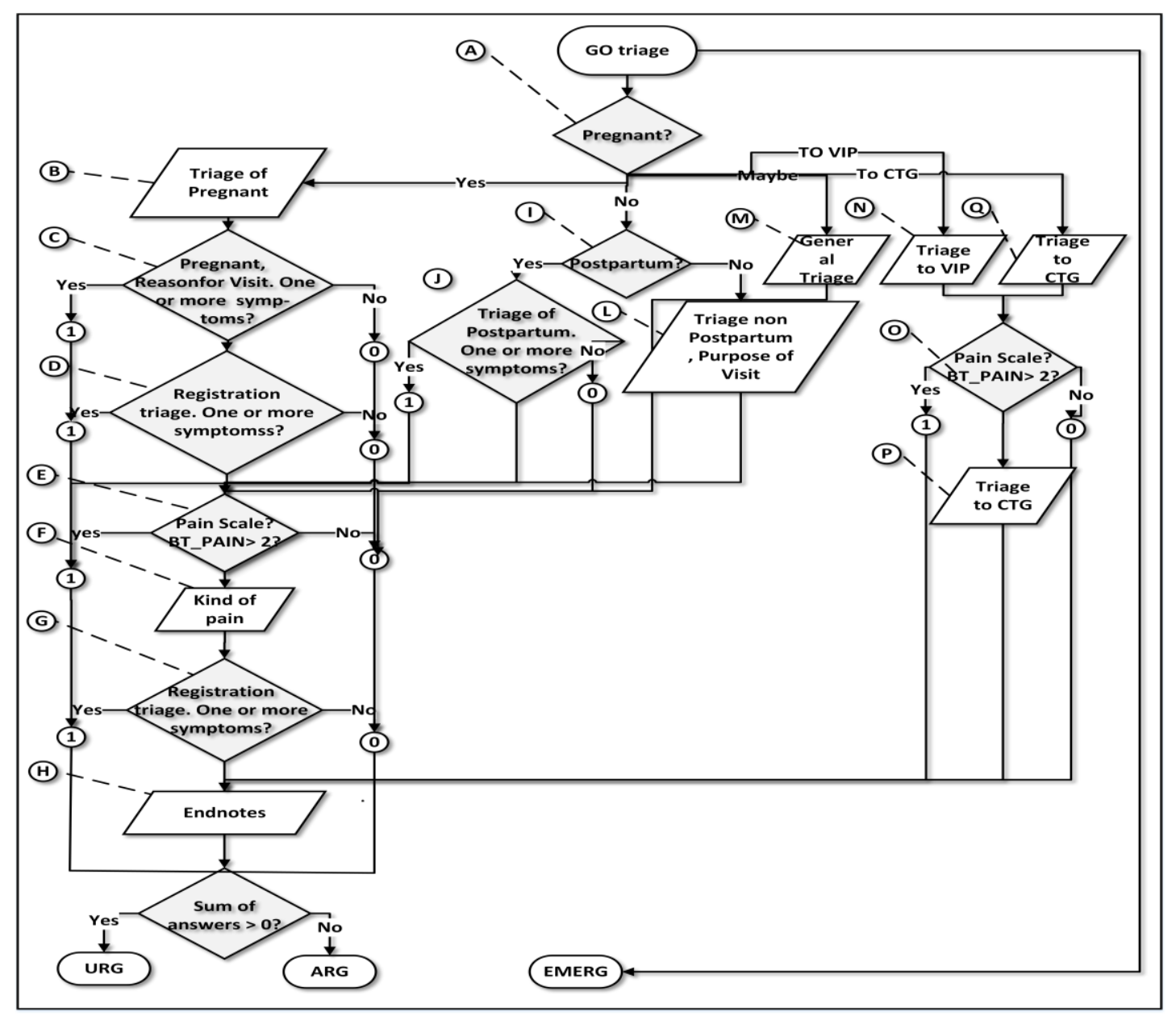

Figure 1. The complete GO workflow.

In the first stage the patients is questioned about if she is pregnant. Given the response, the patient is placed in one of five different situations: (A.1); The patient is pregnant (Yes); (A.2) The patient is not pregnant (No); (A.3) The patient does not know if she is pregnant (Maybe); (A.4) The patient goes to ED of gynecology and obstetrics to do an abortion (To VIP); (A.5) The patient goes to the ED of gynecology and obstetrics to go to CTG ( TO CTG); At this early stage, independently of the patient's response, the patient is identified as ARGO. 


\section{Pregnant woman}

If the patient is identified as pregnant, the system is routed to the page titled Triage of pregnant (Figure 2).

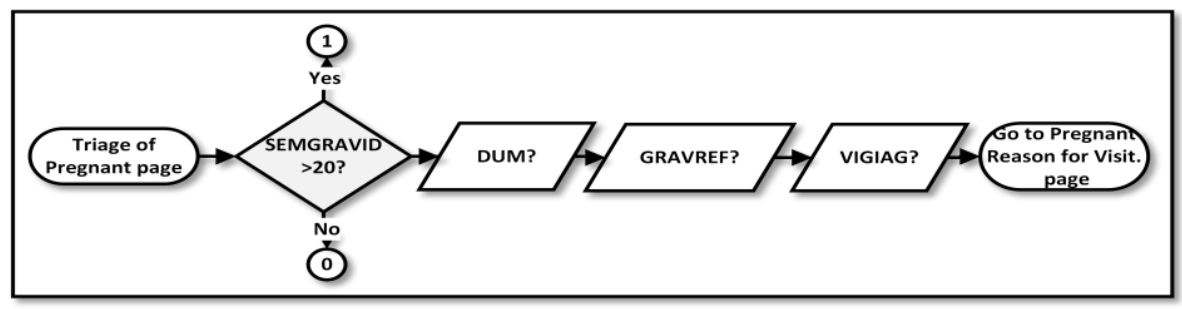

Figure 2. Questions of the page triage of pregnant.

On this page, as noted in the figure 2, are made a set of informative questions like; (B.1) Weeks of Pregnancy? (SEMGRAVID); (B.2) Date of last menstrual period? (DUM); (B.3) Pregnant referenced by other institutions? (GRAVREF); (B.4) Already started Prenatal monitoring? (VIGIAG). If the number of weeks of pregnancy (SEMGRAVID) is greater than 20 weeks, the patient's condition will automatically switch from the ARGO to URG. The other questions are not a mandatory response. Then the system forwards the questionnaire for the page titled Pregnant, reason for the visit (Figure 3).

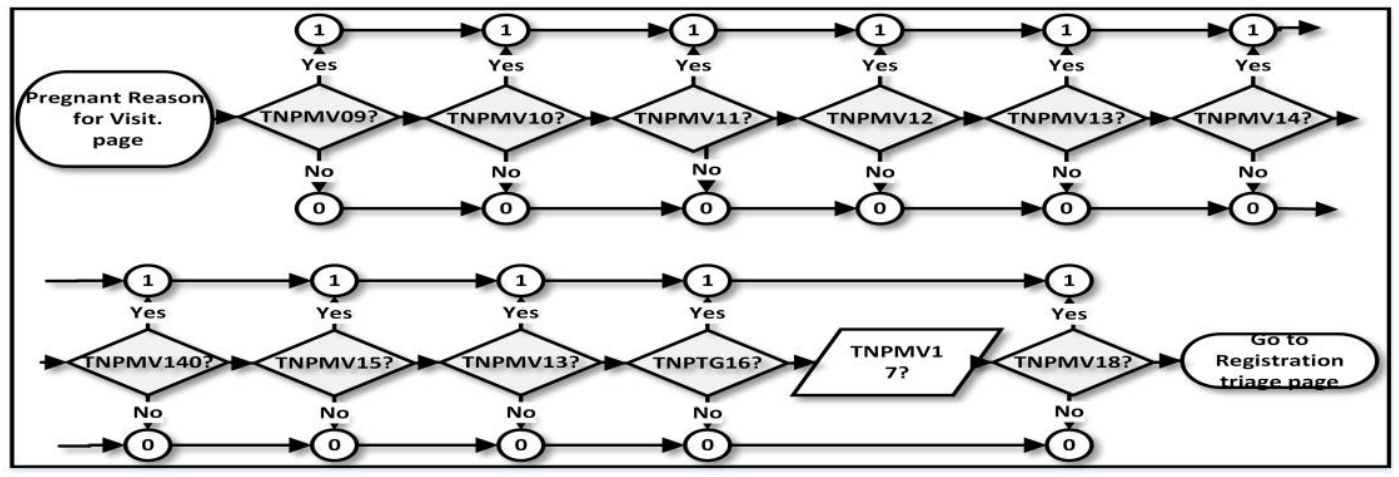

Figure 3. Question of the page pregnant, reason for the visit.

In this page, a set of questions are made in order to find out the reason why pregnant goes to ED of gynecology and obstetrics: (C.1) Headache? (TNPMV09); (C.2) Visual changes? (TNPMV10); (C.3) Reference of tension Increase? (TNPMV11); (C.4) Epigastric pain/right upper quadrant? (TNPMV12); (C.5) Nausea/vomiting? (TNPMV13); (C.6) Changes in skin color/mucous? (TNPMV14); (C.7) Metrorrhagia? (TNPMV140); (C.8) Decreased fetal movements? (TNPMV15); (C.9) Loss of amniotic fluid? (TNPMV16); (C.10) Trauma in Pregnant? (TNPTG16); (C.11) Dating of the pregnancy? (TNPMV17); (C.12) Another pathological reason? (TNPMV18). Except the question TNPMV17, in all the 
others when a question is answered the status changes automatically from ARGO to URG. Then the questionnaire is sent to the page titled Triage registration as showed in Figure 4 .

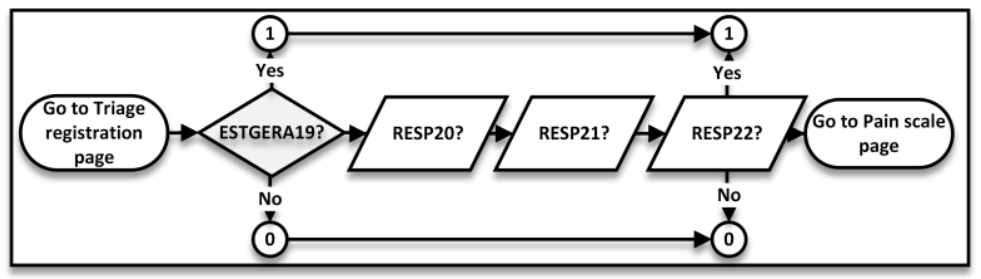

Figure 4. Question of the Triage registration page.

In this section it is discussed some observations and it is evaluated the Glasgow Coma Scale (GCS):

-(D.1) General State? (ESTGERA19), where for this category there are three possible situations are "Good", "Reasonable", "Bad". If the answer is "Bad", the state shall URG, otherwise keeps ARGO;

-(D.2) Ocular Response? (RESP20): In this section are distinguished four situations( No pain, Pain, Verbal Stimulus, Spontaneous)

-(D.3) Verbal Response? (RESP21): In this section five situations are distinguished ( Any response, Incomprehensible, Inappropriate, Confused, and Oriented); -(D.4) Motor response? (RESP22): In the case of motor response six situations (can be identified (Any, Extension to pain, Flexion to pain, Reacts to pain, Find the pain, Obeys orders).

The GCS is a neurological scale that allows you to measure/evaluate the level of consciousness of a person who has suffered a traumatic brain injury. With regard to values, the lowest value that can be obtained with the Glasgow scale is 3 points, while the highest value is 15 points. After these observations the system is routed to the page titled Pain Scale (Figure 5) where assesses the patient's pain.

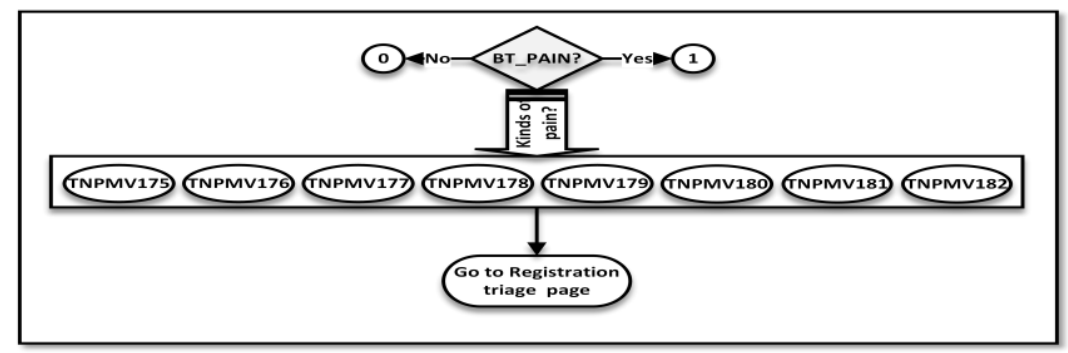


Figure 5. Questions of Pain Scale page.

Depending on this assessment it is assigned a pain value between 1 and 10 . Where 0 represents no pain and 10 is having the most pain. If pregnant present a value greater 0 of pain corresponds to a case of URG otherwise corresponds to ARGO (module E). In this part several hypotheses/painful situations are placed: (F.1) No Pain (TNPMV1720); (F.2) Uterine contractions (TNPMV176); (F.3) Hypogastric pain (TNPMV177); (F.4) Epigastric pain (TNPMV178); (F.5) Iliac Foss Pain (TNPMV179); (F.6) Lumbar Pain (TNPMV180); (F.7) Another Pain (TNPMV181); (F.8) Higher pain 1 week (TNPMV182). In this section it appears that the healthcare professional responsible for triage after observation must check the value of pain and may or may not specify the type of pain. But only the scale parameter of pain allows routing between ARGO or URG. After the questionnaire he is sent to the page titled Registration triage as represented in Figure 6.

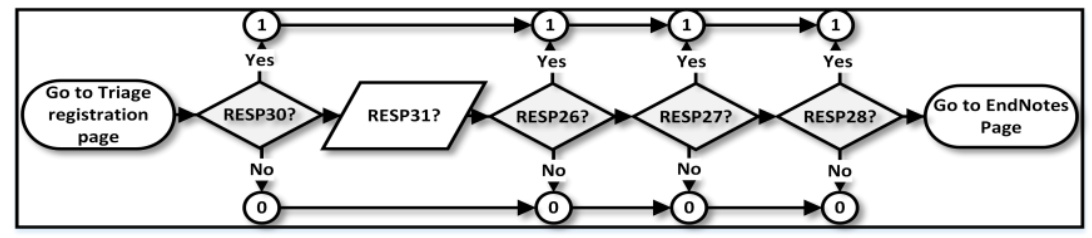

Figure 6. Questions of triage registration page.

This section presents some records about the patient's vital signs. In this section there are the questions where the possible answer can be:

- (G.1) Yes and No and influence the categorization - Up Fever? (RESP25), Urinary symptoms? (RESP26), Convulsions? (RESP28), Syncope? (RESP29). The response options are "yes" and "No". If any of these answers is answered affirmatively is considered a URG case, otherwise it is ARGO.

- (G.2) Yes and No and no influence on categorization - Registration blood pressure? (RESP30) and Combur? (RESP31) - These factors are for information only, not influencing the categorization of the patient.

- (G.3) Multiple choice and influence on categorization - Hemorrhage? (RESP27). The options of choice can be; "Does not have", "Scarce", "Moderate”, and "Severe". If the choice is different of "Does not have" is considered URG. Subsequently the system forwards the questionnaire for the page titled Endnotes (Module $\mathrm{H}$ ), where can be written some relevant free text diagnosis. In the end, the 
system responds with URG, if there is a warning factor being pregnant immediately referred to the emergency department of CMIN, or ARGO was referred for consult.

\section{Not pregnant woman}

If the patient is identified as not pregnant, the system forwards to the questionnaire page titled Triage non-pregnant (Module I) where the patient is asked if she is a postpartum women.

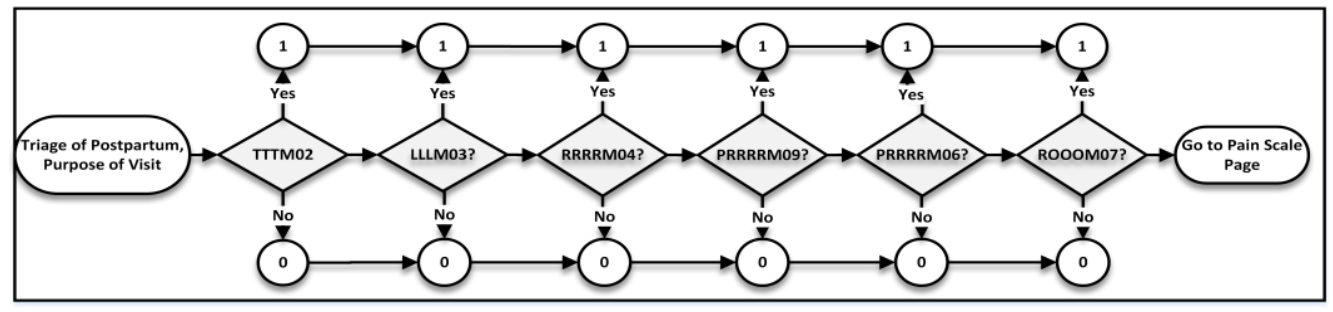

Figure 7. Questions of Triage of Postpartum, Purpose of Visit page.

In the case of it being Postpartum, the system will be sent to the page titled Triage of Postpartum, Purpose of Visit (figure 7) which are made a set of questions to find out the reason for the patient went to ED of gynecology and obstetrics: (J.1) Breast swelling? (TTTM02); (J.2) Fetid lochia? (LLLM03); (J.3) Remove Suture (RRRRM04); (J.4) Fluid (blood or other); (J.5) Passes through the dressing (PRRRRM09); (J.6) Mastitis? (PRRRRM06); (J.7) Another? (ROOOM07). If any of these happen, the patient is categorized as URG otherwise keeps the ARGO state.

Subsequently, the questionnaire is sent to the page titled Pain Scale, represented in the flowchart of Figure 5. In case of she was not postpartum woman, the system will be sent to the page titled Triage non-postpartum, Purpose of Visit (Module L), where they try to find out the reason of the visit to ED of gynecology and obstetrics and subsequently forwarded the questionnaire is also entitled to Pain scale, represented in the flowchart of figure 5 following the normal course, showed in Figure 1.

\section{Maybe pregnant}

If the patient does not know if she is pregnant the system is routed to the page titled General Triage (Figure 8), where the patient is questioned about: (M.1) Date of last menstrual period? (DUM02); (M.2) Reason why she went to ED of gynecology and obstetrics? (MVISTO). 


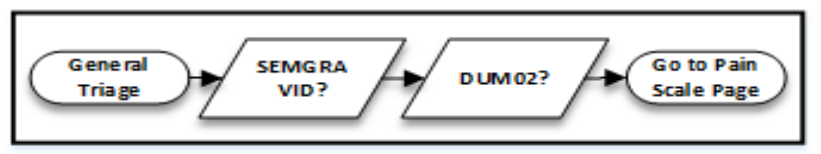

Figure 8. Questions of General Triage page

These questions are for information purposes only. Subsequently the questionnaire is also sent to Pain scale page represented in the flowchart of figure 5 and following the usual course as showed in Figure 1.

\section{Patient to Voluntary Interruption of Pregnancy}

When the patient goes to ED of gynecology and obstetrics to VIP, the questionnaire is sent to the page Triage for VIP (Figure 9), which are made some few Questions/ observations: (N.1) Weeks of pregnancy? (SEMGRAVID); (N.2) Date of last menstruation? (DUM); (N.3) Record blood pressure? (RESP30); (N.4) Combur? (RESP31);

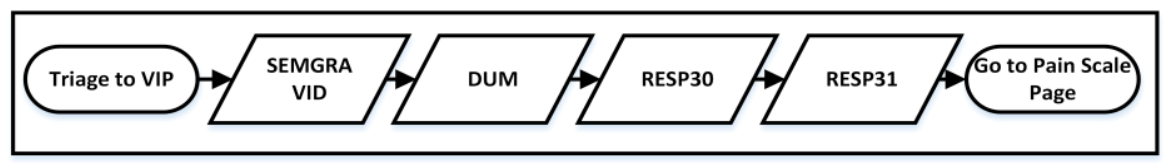

Figure 9. Questions of General Triage page

These questions are only of informative nature. Subsequently the questionnaire is sent to the page titled Pain Scale 1 where it's similar to the described in Figure 5 questionnaire. If the patient experiences any pain this is identified as URG case, otherwise as ARGO. Subsequently, the questionnaire is sent to the final page where can been register some final notes (Endnotes page).

\section{Patient to Cardiotocography}

When the patient goes to ED of gynecology and obstetrics for make Cardiotocography (CTG), the questionnaire is sent to the Triage of Pregnant for CTG page (Figure 10), where some questions/observations are made: (Q.1) Weeks of pregnancy? (SEMGRAVID); (Q.2) Date of last menstruation? (DUM03); (Q.3) Record blood pressure? (RESP30); (Q.4) Combur? (RESP31);

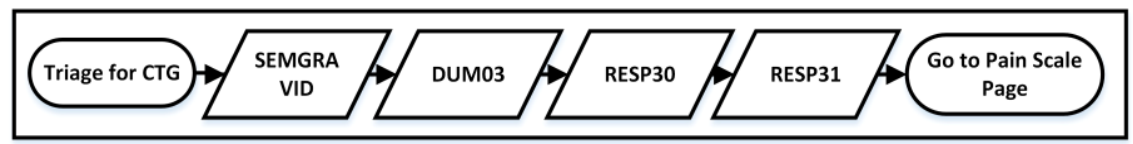

Figure 10. Questions to Triage of Pregnant for CTG.

These issues also are only of informative nature and then the questionnaire is sent to the 
page Pain scale, in the normal course as identified in the figure 5, and then goes to EndNote Page. This is a quick process, only a few important questions will be answered. This process is totally electronic and is supported by the AIDA platform.

\section{Summary of the pre-triage system}

Table 2 present a snapshot of the questions that are asked in the pre-triage system implemented in ED. In this table five aspects are analyzed: The identification of the issue (Group), the expected answer type (Type), the possible response values (Possible values ), defines whether it is a matter of multiple choice or single choice (Choice) and influences or not the issue in determining the categorization of patients $(\mathrm{Dt})$. For a better understanding, some groups of questions were grouped:

- MC - Headache?, Visual changes?, Reference of tension increase?, Epigastric pain/right upper quadrant?, Nausea/vomiting?, Changes in skin color/mucous?, Metrorrhagia?, Decreased fetal movements?, Trauma in Pregnant?, Dating of the pregnancy?, Another pathological reason?;

- $\mathrm{MF}, \mathrm{MP}$ - No Pain, Uterine contractions, Hypo gastric pain, Epigastric pain, Iliac Foss Pain, lumbar Pain, Another Pain, Higher pain 1 week;

- MJ - Breast swelling?, Fetid lochia?, Remove Suture?, Fluid (blood or other)?, Passes through the dressing?, Mastitis?, Another?;

Table 2. Summary of the pre-triage system implemented in to ED.

\begin{tabular}{|l|l|l|l|l|}
\hline Group & Type & Possible Value & Choice & Dt \\
\hline A & Char & $\{$ Yes, No, Maybe, To IGO, To CTG $\}$ & Multiple & No \\
\hline B.1 & Text & $\{0-50\}$ & - & Yes \\
\hline B.2, M.1, & Ate & - & - & No \\
\hline B.3, B.4 N.3, & Integer & $\{$ Yes, No $\}$ & Simple & No \\
\hline C & Char, Text & MC & Multiple & Yes \\
\hline D.1 & Char & $\{$ Good, Reasonable, Bad $\}$ & Simple & Yes \\
\hline D.2 & Char & $\begin{array}{l}\{\text { No pain, Pain, Verbal } \\
\text { Stimulus, Spontaneous }\}\end{array}$ & Simple & No \\
\hline D.3 & Char & $\begin{array}{l}\{\text { Any response, Incomprehensible, } \\
\text { Inappropriate, Confused, Oriented }\}\end{array}$ & Simple & No \\
\hline D.4 & Char & $\begin{array}{l}\{\text { Any, Extension to pain, Flexion to pain, } \\
\text { Reacts to pain, Find the pain, obeys orders }\}\end{array}$ & & No \\
\hline E, O & Interval & {$[1-10]$} & Multiple & Yes \\
\hline F,P & Char, Text & MF, MP & Multiple & No \\
\hline G.1 & Char & $\{$ Yes, No $\}$ & Simple & Yes \\
\hline G.2 & Char & $\{$ Yes, No $\}$ & Simple & No \\
\hline
\end{tabular}




\begin{tabular}{|l|l|l|l|l|}
\hline G.3 & Char & $\{$ Doesn't have, Scarce, Moderate, Severe $\}$ & Simple & Yes \\
\hline H & Text & Free text & - & No \\
\hline I & Integer & $\{$ Yes, No $\}$ & Simple & No \\
\hline J & Chat, Text & $\{$ MJ $\}$ & Multiple & Yes \\
\hline L, M.2 & Text & Motive of the visit? & Figure & No \\
\hline
\end{tabular}

Immediately after the questionnaire be completed, the system will consult a set of models that are suitable for the actual case and try to define the woman condition anticipating the emergency degree of attendance. According to this result, the nurses will decide what to do. In the case of a non-urgent situation an outpatient consult will be schedule. This consult can be of two types: urgent or normal, according to its emergency. The results obtained and decisions made, will be stored for further improvement of the decision models.

\section{Analysis of Pre-Triage System Implementation an CMIN and an Exploration of Possible Developments}

As previously mentioned, the pre-triage system presented in the previous section was implemented at MJD in 2010. Now it is also working in CMIN. This system is the first stage of implementation for a priorities system for ED of Gynecology and Obstetrics. During the last four years, the system has triaged approximately 66730 patients: 18773 in 2010, 18348 in 2011, and 7445 in 2012 and 17164 patients in 2013. As stated in the previous section, this system consists of six flowcharts matching the six patient's observed in CMIN. Statistically can be said that in these four years were cared about: 35238 pregnant women; 4050 to postpartum woman; 24547 to non-postpartum and nonpregnant woman; 4754 to patients who may be pregnant; 2843 to CMIN patients who use the process to make the Voluntary Interruption of Pregnancy (VIP); 2511 patients to Cardiotocography (CTG). An analysis was also performed in terms of the number of patients who were distinguished as URG or ARGO, for all classes of patients (Table 3).

Table 3. Statistical analysis of the number of patients triaged by CMIN pre-triage system distributed flowcharts/patient types and the resulting pre-triage system.

\begin{tabular}{|c|c|c|c|}
\hline Designation & $\begin{array}{c}\text { Total for pre- } \\
\text { triage result }\end{array}$ & Total & Percentage \\
\hline
\end{tabular}




\begin{tabular}{|l|r|r|r|}
\hline ARGO pregnant woman & 13117 & 35225 & $37.24 \%$ \\
\hline URG pregnant woman & 22108 & 35225 & $62.76 \%$ \\
\hline ARGO postpartum woman & 1634 & 4051 & $40.34 \%$ \\
\hline URG postpartum woman & 2417 & 4051 & $59.66 \%$ \\
\hline ARGO non-postpartum woman & 16481 & 24538 & $67.17 \%$ \\
\hline URG non-postpartum woman & 8057 & 24538 & $32.83 \%$ \\
\hline ARGO maybe pregnant & 3605 & 4753 & $75.85 \%$ \\
\hline URG maybe pregnant & 1148 & 4753 & $24.15 \%$ \\
\hline ARGO to VIP & 2761 & 2843 & $97.12 \%$ \\
\hline URG to VIP & 82 & 2843 & $2.88 \%$ \\
\hline ARGO to CTG & 82 & 611 & $13.42 \%$ \\
\hline URG to CTG & 529 & 611 & $86.58 \%$ \\
\hline
\end{tabular}

In order to understanding the waiting time improvements in case of URG and ARGO, was done a study filtering all cases with a waiting time between 0 and 360 minutes and a triage date between $1^{\text {st }}$ January 2010 and $21^{\text {st }}$ December 2013. They were analyzed approximately 62576 cases. The statistical analysis for each level of pre-triage is shown in Table 4. For example the average waiting time to the URG level is 6 minutes and 55 seconds, being the mode around 4 minutes.

Table 4 - Statistical analysis of CMIN pre-triage system waiting time.

\begin{tabular}{|r|r|r|r|r|r|r|r|}
\hline Level & $\begin{array}{c}\text { Number of } \\
\text { patients }\end{array}$ & Average & $\begin{array}{c}\text { Standard } \\
\text { Deviation }\end{array}$ & Mode & Median & Minimum & Maximum \\
\hline URG & 29137 & $00: 06: 55$ & $00: 16: 37$ & $00: 04: 00$ & $00: 04: 00$ & $00: 00: 00$ & $05: 45: 36$ \\
\hline ARGO & 33439 & $00: 07: 17$ & $00: 15: 05$ & $00: 03: 00$ & $00: 05: 00$ & $00: 00: 00$ & $05: 39: 00$ \\
\hline
\end{tabular}

Using this pre-triage system, urgent cases (more urgent), take less time to be attended, on average, than cases identified as ARGO (less urgent). In this particular case the first phase of implementation of the priorities system is already operating in CMIN and in four years of existence has been shown that this system has the ability to triage patients into two levels. In this respect some researches has been made based on the MTS, to be a standard at national and European level as regards the general triage and very well known by health professionals of the national health system; the triage system OTAS, being a worldwide reference in the specific cases of gynecologists, and CMIN pre-triage system described in this article. Still to be that this pre-triage system already implemented enables a more practical view of what will be a future system specific priorities for GO. Among other system features the fact of it been supported by AIDA, make it permanently online and presenting characteristic of eHealth. It is able to ensure 
the interoperability between AIDA, the SAPE, and SAM, and the remaining CHP information systems. This feature will certainly be present at a later stage of the evolution of this system. Moreover, this pre-triage system already be familiar to CMIN health professionals is an asset to the extent that enable better adaptation to new developments.

\section{Discussion and Conclusion}

This work has emerged due to some inefficiencies found in the MTS who is working in the $\mathrm{CHP}$ when applied to women who seek emergency care for genecology and obstetrics. After a quick study it was possible observe that this inefficiency is transversal to other hospitals and countries. The MTS system in question is not prepared to Maternity Care particularities due to the generality of the questions used in triage process. The gaps was found when the system it was used to triage cases of gynecology and obstetrics and the results presented an excess of urgent cases, i.e., a high number of false positives. This situation lead to a need of building a new system (pre-triage) to bring some balance between the urgent and non-urgent cases and a better patient categorization. This pre-triage system developed, shown to be most balanced when applied in the MJD / CMIN emergency. Regarding the development of the pretriage system presented in this article, it was based on some of the MTS issues but with different levels of response - appropriate for the relevant needs of Maternity Care. The structure of the decision model was inspired in the MTS flowcharts because it is divide by symptoms / reasons (pregnancy, asthma, etc.) and in the case of pre-triage system the division was made by class of patients (pregnant, postpartum, non-postpartum, maybe pregnant, for Voluntary Interruption of Pregnancy (VIP) or for Cardiotocography (CTG)). The model is composed by a set of symptoms, and the decision methodology is similar to the MTS, only instead of presenting 5 levels of priority at this early stage it was decided to only define two classes of distinction (for a safety issue / need and assess if the system and the methodology adopted would be reliable). To assess the quality improvement and the evolution of the pre-triage system over the past four years it was evaluated the attained results with the waiting 
time for the levels of URG and ARGO. To perform this analysis (Table 5) they were compared the values presented in Table 1 (T1) - waiting time of 2010 with the results presented in Table 4 (T4) waiting time for the years 2010, 2011, 2012 and 2013.

Table 5 - Waiting time differences (2010 to 2013)

\begin{tabular}{|r|c|c|c|c|}
\hline \multicolumn{1}{|c|}{ Level } & $\begin{array}{c}\text { Average Time } \\
(\mathrm{T} 4-\mathrm{T} 1)\end{array}$ & $\begin{array}{c}\text { Standard Deviation } \\
(\mathrm{T} 4-\mathrm{T} 1)\end{array}$ & $\begin{array}{c}\text { Mode } \\
(\mathrm{T} 4-\mathrm{T} 1)\end{array}$ & $\begin{array}{c}\text { Median } \\
(\mathrm{T} 4-\mathrm{T} 1)\end{array}$ \\
\hline URG & $00: 17: 45$ & $00: 05: 31$ & $00: 04: 20$ & $00: 13: 07$ \\
\hline ARGO & $00: 17: 06$ & $00: 05: 45$ & $00: 00: 43$ & $00: 11: 01$ \\
\hline
\end{tabular}

When analyzed the results presented in the Table 5 it is possible verify a noteworthy decreasing in the waiting time. In the case of average waiting time, the differences are in both cases near 17 minutes. These values mean that between 2013 and 2010 the waiting time decreased in around 17 minutes. In terms of standard deviation, it also had an important decrease. In the case of URG it was found a decreased in 5 minutes and 31 seconds and in the ARGO cases the deviation decreased in 5 minutes and 42 seconds. The same type of analysis can be done to the other metrics: mode and median. The minimum and maximum times are similar. This happens because the emergent cases are immediately cared and there are some other patients that the care are minimum and do not needed an urgent attention. To all the cases it is possible to verify better results (lower waiting times) now than in 2010. With the introduction of the pre-triage system the waiting time for the two levels tends to be increasingly closer to the expected average time. As the average waiting time decreased significantly in the last four years compared when compared with 2010, it can be concluded that the waiting time with this system tends to decrease for the two levels in study, providing a better, efficiently and quality service. Other possible analysis is study the waiting time by level and year. In 2010 the average waiting time was slightly higher for the URG (0:24:40) cases then ARGO (00:24:23) cases. These times proved the need of having a specialized triage system because it gave a wrong significance of the emergent concept. In 2010 patients with less care (ARGO) are cared most quickly. In the analysis of the last 4 years have seen the opposite situation, the times were corrected and are now adequate. The average waiting time for the ARGO case (00:07:17) is slightly higher than URG (00:06:55). Being the URG the highest priority level, it is possible concluded that this pre-triage system is 
calibrated to triage patients at two levels of priority, giving most importance to the urgent cases. Finally this article presented new triage model specific for gynecology and obstetrics. Scientifically the workflow and questionnaire presented brings advantages to the medical and scientific communities because it constitutes a starting point for the implementation of a specific priority triage system for GO on national and international that support Maternity Care institutions.

\section{Future Work}

The pre-triage system described in this article and correctly implemented in CMIN has many potentialities. One of the great potential of this system is the possibility of transforming an existing system similar to the system priorities MTS, but specific for GO. Thus, each color would be associated with a time limit of waiting, as the gravity of the patient (5 levels of priority: Emergent, Very Urgent, Urgent system not urgent, not urgent). The only difference lies in the triage questionnaire, in which is already implemented, that is more specific to the type of users of maternity care. The next step consists in convert this pre-triage system into a complete five levels system. This process requires the creation of a multi-area group (informatics and health) and several months of model / system optimization and field tests. In addition, another major potential is implementing a Business Intelligence platform in which, through the data recorded in the Triage System would make it possible to obtain indicators which reflect the operation of CMIN, enabling process improvement.

\section{Acknowledgements}

"This work is funded by National Funds through the FCT - Fundação para a Ciência e a Tecnologia (Portuguese Foundation for Science and Technology) within projects PEstOE/EEI/UI0752/2014 and PEst-OE/EEI/UI0319/2014" 


\section{References}

Abelha A., Machado J., Santos M., Allegro S., Rua F., Paiva M. \& Neves J. (2003). Agency for Integration, Diffusion and Archive of Medical Information, in Proceedings of the Third IASTED International Conference - Artificial Intelligence and Applications(ISBN 0-88986-390-3), Malaga, Spain, 2003.

Beveridge R. (1998). The Canadian Triage and Acuity Scale: a new and critical element in health care reform. Canadian Association of Emergency Physicians. J Emerg Med 1998;16(3):507-11.

Cabral, A., Pina, C., Machado, H., Abelha, A. ,Salazar, M., Quintas, C., Portela, F, Machado, J., Neves, J. \& Santos, M., (2011) Data Acquisition Process for an Intelligent Decision Support in Gynecology and Obstetrics Emergency Triage, in Communications in Computer and Information Science, 221 CCIS (PART 3), Springer.

Cardoso, L.; Marins, F.; Portela, F.; Santos, M.; Abelha, A. \& Machado, J. (2014).. The Next Generation of Interoperability Agents in Healthcare. Int. J. Environ. Res. Public Health 11, no. 5: 5349-5371, MDPI, 2014. Retireved from http:/ / www.mdpi.com/1660-4601/11/5/5349

Machado J., Alves V., Abelha A. \& Neves J. (2007). Ambient Intelligence via Multiagent Systems in Medical arena; International Journal of Engineering Intelligent Systems, Special issue on Decision Support Systems; vol. 15, n.3, pp. 167-173.

Mackway-Jones, K. (1996). Emergency Triage: Manchester Triage Group. Wiley. Retrieved from http://books.google.co.in/books?id=xEQIGwAACAAJ

Moll, H. A. (2009). Challenges in the validation of triage systems at emergency departments. Journal of clinical epidemiology.

Murray, M., Bullard, M., \& Grafstein, E. (2004). Revisions to the Canadian Emergency Department Triage and Acuity Scale implementation guidelines. 
Cjem, 6(6), 421-7. Retrieved from

http://www.ncbi.nlm.nih.gov/pubmed/17378961

Portela, F., Cabral, A., Abelha, A., Salazar, M., Quintas, C., Machado, J., Neves, J. \& Santos,M.. (2013a). Knowledge Acquisition Process for Intelligent Decision Support in Critical Health Care. In Information Systems and Technologies for Enhancing Health and Social Care. Page:55-68 ISBN13: 9781466636675. IGI Global.

Portela F., Gago P., Santos M., Machado J., Abelha A., Silva A., Rua F., Quintas C.\& Pinto F. (2013b). Implementing a Pervasive Real-time Intelligent System for Tracking Critical Events with Intensive Care Patients. IJHISI International Journal of Healthcare Information Systems and Informatics, Volume 8, Issue 4, IGI Global.

Portela F., Santos F., Silva A., Machado J.,Abelha A. and Rua F. (2014). Pervasive and Intelligent Decision Support in Intensive Medicine - The Complete Picture . Lecture Notes in Computer Science (LNCS) - Information Technology in Bio- and Medical Informatics. Springer.

Smithson, D. S., Twohey, R., Rice, T., Watts, N., Fernandes, C. M., \& Gratton, R. J. (2013). Implementing an obstetric triage acuity scale: interrater reliability and patient flow analysis. American Journal of Obstetrics and Gynecology, 209(4), 287-93. doi:10.1016/j.ajog.2013.03.031

Turban, E., Aronson, J. E., \& Liang, T.-P. (2004). Decision Support Systems and Intelligent Systems (7th Edition). Upper Saddle River, NJ, USA: Prentice-Hall, Inc. 